\title{
Facilitating Minority Medical Education, Research, and Faculty
}

\author{
John M. Carethers ${ }^{1}$
}

Published online: 8 February 2016

(C) Springer Science+Business Media New York 2016

The value of having a diverse physician and physicianscientist workforce is incalculable for the USA. There is no substitute for having a physician workforce with various backgrounds in patient care and research. New clinicians develop lifelong colleagues and relationships that take into account each other's opinion and experiences, and scientists consider novel or innovative opinions for approaches to solving a research question. The value of diversity in American medicine, from the pre-Civil War era to the modern era, has been understated with regard to the involvement of underrepresented minorities (URMs) in the many successes that the country has had in the health sciences-from drug and device development to basic research that ultimately leads to a breakthrough. This is despite the challenges that prevent many URMs and other groups from having the opportunity for a career in the medical sciences, as well as from succeeding once an opportunity presents itself. There are likely multiple "diamonds in the rough" in communities or URM families who do not have the resources to allow consideration of a career in the medical sciences. Some of this failure is attributable to socioeconomic issues, the limitations of local schools, constrained expectations for an individual's potential, lack of exposure to these types of careers, and the early initiation of bias by society owing to an individual's skin color or ethnicity.

John M. Carethers

jcarethe@umich.edu

1 Division of Gastroenterology, Department of Internal Medicine, University of Michigan, 3101 Taubman Center, 1500 East Medical Center Drive, Ann Arbor, MI 48109-5368, USA
With the predicted change in the US census to a nonmajority population by 2060 , and URMs comprising $57 \%$ of the US population by then [1], a path should be laid out to lower the barriers to URMs for entering and succeeding within the medical sciences to approximate their representation in the population. Some patients prefer to be examined by a physician with a similar background, but the number of URMs in minority education remains small and is not reflective of the US population as a whole [2], thereby limiting the number of these physicians to care for the expanding URM population. The "pipeline" of URMs entering medical schools has grown slowly-to about 2500 students nationwide out of $\sim 20,000$ total medical school matriculants [2]. This rate will not match the nation's need for a diverse medical work force; only $\sim 7 \%$ of all physicians are from URM groups. Even more concerning is the low number of URMs entering and staying in academic medicine. Only a fraction (perhaps $10-15 \%$ or less) of those matriculating URM medical students will eventually engage fully in the traditional academic tripartite mission, making the pipeline to academic medicine for URMs only a trickle. Similarly, medical school faculty makeup does not reflect the US population, with URMs representing $\sim 7 \%$ of faculty [2]. The dearth of URM medical faculty members is a lost opportunity to provide role models and mentors for URM students, not to mention representation on student and faculty selection committees that provide complete views for selection and hiring of a diverse student body and faculty.

Once on faculty, URMs face continued challenges to success. First, with few other URM faculty, they are often asked to be on multiple committees for representation purposes, potentially slowing down their academic progress. Second, they are often expected to be the URM face for any URM student who comes through. Being a role 
model is good, but it can be bad if there is a high URM student-to-faculty ratio. Third, evidence suggests that for URM researchers, particularly African American researchers, the success of obtaining an NIH R01 grantthe bread and butter of independent research and often a determinant for academic promotion-is at least $10 \%$ less for this group than for their non-URM counterparts [3].

\section{Approaches to Dealing with the Pipeline and Retention of URM Medical Professionals}

Some approaches have been suggested to help rectify the disparity in our medical workforce. The pipeline issue is a significant and challenging one. One approach taken at the University of California, San Diego, was to create a 6ththrough 12th-grade charter school that targets economically disadvantaged students to prepare them for entry into the University of California system. The Preuss School has been highly successful, with a college matriculation rate of $>90 \%,>70 \%$ of whom are URMs [4]. These students can choose any career once they are in college, and some choose medical careers. Preparing students for medical school once in college can be an intervening point. Some schools such as Wayne State University under the late Charles Whitten developed a postbacculaureate program that helps prepare students for the rigors of medical school [5], and other schools have initiated similar programs. Once in medical school, enrichment of a diverse student body is typically individualized by each medical school, and the Liaison Committee on Medical Education (LCME) and American Association of Medical Colleges (AAMC) have made diversity a stated priority [6].

There is no doubt that the drive of the student must be front and center as a determinant in earning a diploma. However, several circumstances should be considered by medical schools to help ensure that their graduation rates for URMs match their matriculation numbers. These include social issues: Some students help to care for extended family members or provide financial support to the family; access to a connected set of mentors and advisors with a college degree or medical background may be limited; vigorous study habits and tutorials may not have been experienced in their prior education; roles models for success are limited; and advocacy from segments of the faculty may be limited.

The road to an academic career is even more bumpy because this type of career is considered only by a small fraction of URMs who go through medical training. Several programs have been developed because of the absolute need to attract URMs into academic medicine. Without these programs, percentages would likely be much worse than the already overall low numbers of URMs considering a career in academics. At the high school and college levels, the National Institute of Diabetes and Digestive and Kidney Diseases (NIDDK) Short-Term Education Program for Underrepresented Persons (STEP-UP) provides 12 weeks of laboratory exposure with an established investigator and exposure to the National Institutes of Health (NIH) campus in Bethesda, Maryland, at the end of the program [7]. At the college level, NIH/National Institute of General Medical Sciences (NIGMS) programs such as the Minority Biomedical Research Support (MBRS) and the Minority Access to Research Careers (MARC) programs provide predoctoral exposure and training through participating laboratories. The American Gastroenterological Association (AGA) established an NIH R25 training program for URMs at the college or medical school level for exposure to gastroenterology research, as have some other organizations. At the college, medical, predoctoral, or postdoctoral levels, the $\mathrm{NIH}$ will evaluate minority supplement applications to holders of $\mathrm{R}, \mathrm{U}$, and $\mathrm{T}$ grants, which can provide up to 2-3 years of support. At the resident or fellow stage, traditional T32 or National Research Service Award (NRSA) support is often critical to jump start a research career, whether or not an individual is a URM. At the faculty level, there are fewer programs specific to URMs. Junior faculty can compete for the Harold Amos Faculty Development Program sponsored by the Robert Wood Johnson Foundation, an outstanding program that boasts a high success rate for academic success for URMs, including many who have obtained national leadership positions. This program requires a mentor who also participates in the program. Of course, none of these programs matters if the URM is not aware of their existence or if potential mentors do not connect with them as mentees.

Lastly, while the aforementioned programs are likely beneficial, the onus is on all of us in the profession to help achieve a diverse workforce. Once on faculty, a URM faces the same challenges as any other faculty [8], but challenges could be amplified by: (1) lack of role models with similar backgrounds, (2) lack of mentorship to navigate promotion timelines and milestones, and (3) challenges for funding at the national level [3]. Some medical schools help guide all faculty and may pay special attention to URM faculty, but this is highly individualized at each school, with many schools likely not paying attention to it.

\section{A Twist in My Own Career}

I have been fortunate to have had a drive for good performance, great mentorship, and having others see my potential to take me to the next level. I had a supportive family, participated in Wayne State's postbacculaureate program at the start of medical school, was on T32 and 
NRSA grants, and obtained a Robert Wood Johnson Harold Amos Award as a young faculty member. My research mentor made me a part of his laboratory team without any reservation and to this day still provides career advice to me. I provided mentorship to Minority Biomedical Research Support (MBRS), Minority Access to Research Centers (MARC), and McNair program students, as well as to many URM students who are not part of those programs. Opportunities presented themselves as I progressed through the ranks in academia. The interesting thing is that with my training in colorectal cancer genetics, I began to explore some unanswered questions about the biology of colorectal cancer disparities, something I did not initially focus on when I began my laboratory training. Because of this new interest and my background (and perhaps less interest by others with similar training but different backgrounds), I realized over time that I might be one of a handful of investigators who could actually explore this area in a unique way. This was a relatively distinctive endeavor, but with my particular circumstances, background, and training, these were some of the questions that needed to be addressed and otherwise might not have been. This new interest has led to several review articles, opinion pieces, and research manuscripts that have examined this disparity [9-13]. Additionally, it has led to NIH funding through NIH U and R mechanisms for my laboratory and generated interest from URM students to participate in research. My foray into this area was not predictable when I started out; it grew out of my background combined with my original interests and developed synergistically by circumstance. Additionally, the research area has amplified participation in research by URM students. My example could be replicated in many forms when opportunities exist for URMs. The investment made for my development in science [14] has not only been wonderful for my career, but has also developed me as a role model, mentor, advisor, investigator, and leader for the next generation, as well as stimulating interest in medical research by the next generation.

\section{What You Can Do as a Medical Professional}

1. Be a mentor and advisor to a URM at any stage- - high school, undergraduate, medical student, resident, fellow, or faculty.

2. Be a role model to a URM. My own research mentor was not from the same racial background as me, and, conversely, I have mentored many students and trainees with racial backgrounds different from my own. The important thing is to show a mentee that being a physician or physician-scientist is important, fun, and engaging. It is a career path that anyone of any background can succeed in.

3. Invite URM students to experience your research program or expose them to clinical medicine. This could be something as simple as a conversation, sharing an article, touring a research laboratory, or observing you in the care of patients.

4. Participate with students from MBRS, MARC, and R25 programs if they are available in your area. It may make the difference in developing interest in research for a student.

5. Make URMs aware of various programs mentioned in this article, and any others that might be helpful, to develop or retain them as medical professionals.

6. Pay attention to URM faculty and help guide and advise them through the milestones needed for promotion and throughout their careers.

7. Be mindful of the makeup of selection committees, question whether the committee has the right representation, and be objective about the selection of students and faculty.

Overall, a diverse medical specialty is a strong, innovative, and creative medical specialty.

Acknowledgments I am supported by the United States Public Health Service (DK067287 and CA162147) and the A. Alfred Taubman Medical Research Institute of the University of Michigan.

\section{References}

1. https://www.census.gov/newsroom/releases/archives/population/ cb12-243.html.

2. Merchant JL, Omary MB. Underrepresentation of underrepresented minorities in academic medicine: The need to enhance the pipeline and the pipe. Gastroenterology. 2010;138:19-26.

3. Ginther DK, Schaffer WT, Schnell J, et al. Race, ethnicity, and NIH research awards. Science. 2011;333:1015-1019.

4. https://preuss.ucsd.edu.

5. http://diversity.med.wayne.edu/postbac.php.

6. www.paeaonline.org/index.php?ht=a/GetDocumentAction/ i/73942.

7. http://www.niddk.nih.gov/research-funding/process/diversity/ research-and-training-for-students/short-term-research-experi ence-underrepresented-persons/Pages/default.aspx.

8. Carethers JM. Starting your first faculty position. Gastrointest Endosc. 2007;66:1186-1187.

9. Carethers JM. Racial and ethnic factors in the genetic pathogenesis of colorectal cancer. J Assoc Acad Minor Phys. 1999;10:59-67.

10. Ayanian JZ, Carethers JM. Bridging behavior and biology to reduce socioeconomic disparities in colorectal cancer risk. J Natl Cancer Inst. 2012;104:1343-1344.

11. Carethers JM, Murali B, Yang B, et al. Influence of race on microsatellite instability and $\mathrm{CD} 8^{+} \mathrm{T}$ cell infiltration in colon cancer. PLoS One. 2014;9:e100461.

12. Carethers JM. Screening for colorectal cancer in African Americans: Determinants and rationale for an earlier age to commence screening. Dig Dis Sci. 2015;60:711-721. 
13. Kupfer SS, Carr RM, Carethers JM. Reducing colorectal cancer risk among African Americans. Gastroenterology. 2015;149: 1302-1304.
14. Carethers JM, Coughlin S, Diamond B, et al. AAP position statement: The imperative to invest in science has never been greater. J Clin Invest. 2014;124:3680-3681. 